\title{
A SELF-CALIBRATING MULTICOMPONENT FORCE/TORQUE MEASURING SYSTEM
}

\author{
Rafael R. Marangoni ${ }^{1}$, Jan Schleichert ${ }^{1}$, Ilko Rahneberg ${ }^{2}$, \\ Falko Hilbrunner ${ }^{3}$ and Thomas Fröhlich ${ }^{1}$
}
${ }^{1} \mathrm{TU}$ Ilmenau, Institute for Process Measurement and Sensor Technology, Ilmenau, Germany
${ }^{2}$ SIOS Meßtechnik GmbH, Research \& Development, Ilmenau, Germany
${ }^{3}$ Sartorius Lab Instruments GmbH \& Co. KG, Research \& Development Mass Comparators, Göttingen, Germany

\begin{abstract}
A multi-component self-calibrating force and torque sensor is presented. In this system, the principle of a Kibble balance is adapted for the traceable force and torque measurement in three orthogonal directions. The system has two operating modes: the velocity mode and the force/torque sensing mode. In the velocity mode, the calibration of the sensor is performed, while in the force/torque sensing mode, forces and torques are measured by using the principle of the electromagnetic force compensation. Details about the system are provided, with the main components of the sensor and a description of the operational procedure. A prototype of the system is currently being implemented for measuring forces and torques in a range of $\pm 2 \mathrm{~N}$ and $\pm 0,1 \mathrm{Nm}$ respectively. A maximal relative expanded measurement uncertainty $(\mathrm{k}=2)$ of $10 \cdot 10^{-5}$ is expected for the force and torque measurements.
\end{abstract}

Index Terms - Force metrology, torque metrology, multi-component, traceable measurement, Kibble balance, calibration

\section{INTRODUCTION}

Several calibration setups for multi component force and torque sensors have been presented in the literature. For instance, the Physikalisch-Technische Bundesanstalt (PTB) in Germany have two calibration setups [7,12] used for calibrating multi-component force sensors. The first system can be used to calibrate six component force and torque sensors and has a measuring range of up to $10 \mathrm{kN}$ for the forces and $1 \mathrm{kN} \cdot \mathrm{m}$ for the torques. With this system it is possible to measure forces and torques with a relative expanded measurement uncertainty $(\mathrm{k}=2)$ of $2,2 \cdot 10^{-4}$. The second system is a deadweight force/torque standard machine and can be used to calibrate two-component systems. It has a range from $20 \mathrm{kN}$ to $1 \mathrm{MN}$ for the force component and $20 \mathrm{~N} \cdot \mathrm{m}$ to $2 \mathrm{kN} \cdot \mathrm{m}$ for the torque component. Forces and torques with an expanded relative uncertainty $(\mathrm{k}=2)$ of respectively $2 \cdot 10^{-5}$ and $3,9 \cdot 10^{-4}$ can be measured. Other works have addressed the calibration of multi-component force and torque sensors in different measuring ranges. For instance, Ferrero et al [2] have developed a six-component system that operates with forces up to $105 \mathrm{kN}$ and torques up to $2 \mathrm{kN} \cdot \mathrm{m}$. Kim et al [6] have developed a system that generates forces and torques in ranges of $50 \mathrm{~N}$ to $500 \mathrm{~N}$ and $5 \mathrm{~N} \cdot \mathrm{m}$ to $50 \mathrm{~N} \cdot \mathrm{m}$ respectively. For forces and torques in the range of $2 \mathrm{~N}$ and $0,15 \mathrm{~N} \cdot \mathrm{m}$ a calibration setup was designed in the Technische Universität Ilmenau [8]. With this system it is possible to determine the calibration coefficient for the sensors with a relative expanded uncertainty $(\mathrm{k}=2)$ of $5 \cdot 10^{-3}$. A common fact for all systems mentioned here is that the forces and torques are realized either by deadweights or by calibrated force/torque sensors. For the systems based on deadweights there is an 


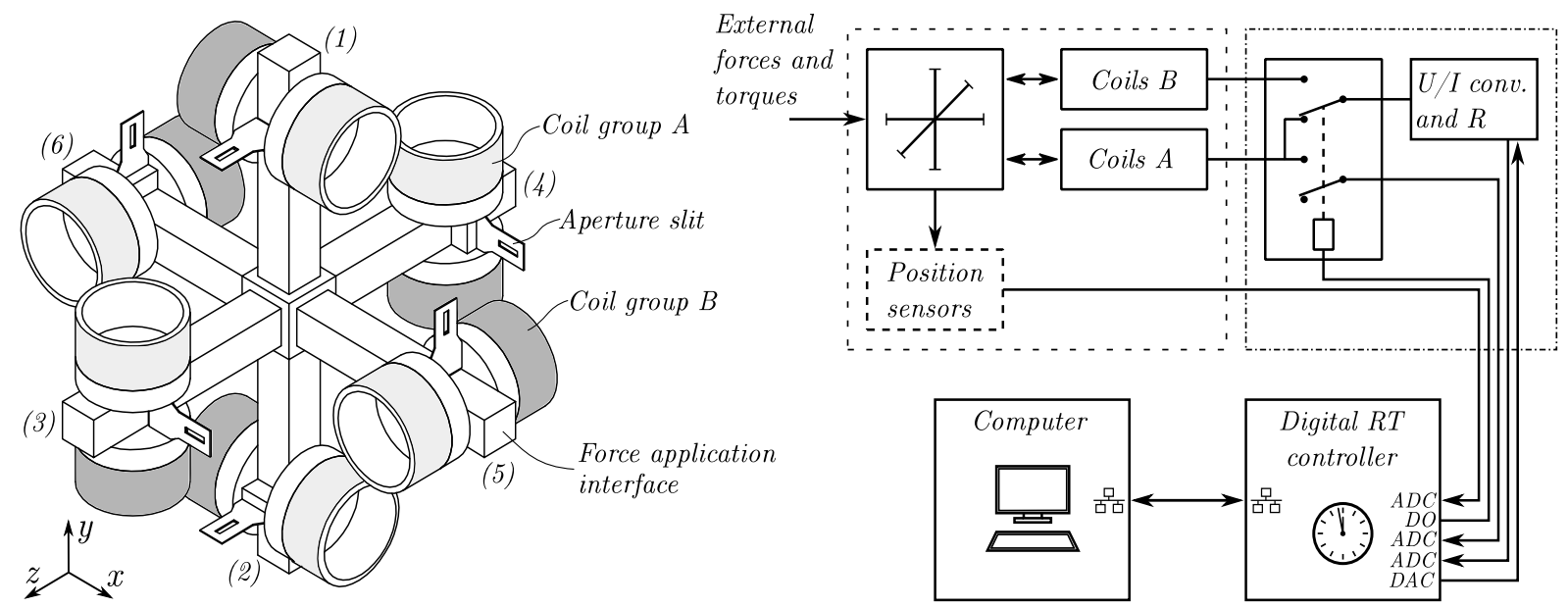

Figure 1: The free flying sensing element on the left hand side and the system plan on the right hand side

additional difficulty to apply horizontal forces, which are originally generated in the vertical i.e. gravity acceleration direction. In this case, it is necessary to use additional mechanisms with levers, bearings or pulleys, to conduct the forces, or rotate the force sensor in order to align the direction to be calibrated with the gravity direction. It is possible to overcome these difficulties by using calibrated force/torque sensors as measurement standards, which can operate in any orientation. However, these force and torque sensors are usually calibrated by using deadweight machines.

In this paper, a self-calibrating multi-component force/torque measuring system based on the Kibble balance [3] is introduced. The Kibble balance is currently being used for the redefinition of the kilogram in the revised International System of Units $[4,11]$. The system presented here is a realization of the principle first described in [13] and includes references for position, angle, voltage, resistance and time, which are used to provide traceable measurements for the calibration factors of the sensor. In this way, calibration methods only based on electrical, time, length and angle measurements are necessary. So, the use of deadweights and the gravitational acceleration $g$ are avoided. It is possible to use this system as a fundamental force and torque measurement standard, to calibrate other sensors, and also to measure forces and torques in different applications, providing a traceable measurement with improved uncertainty through in-process calibration. Furthermore, since the system is also based on the principle of the electromagnetic force compensation (EMFC), other advantages are included: there is no displacement of the sensing element during the measuring process and nonlinearities in material deformations are avoided [5,10]. The system presented here has not only the potential to simplify the traceable measurement of forces and torques, but also to improve the measurement uncertainty in the force/torque range covered by this system.

\section{DESCRIPTION OF THE SYSTEM}

The main component of the system is a free flying servo controlled element with six position sensors and twelve voice coil actuators attached to it (figure 1, left hand side). The voice coil actuators are divided in two groups with six actuators each. We name them A coils and B coils in the following. The system has also two operating modes, called the velocity mode and the force/torque sensing mode. By using a multi-input multi-output (MIMO) control system, the signals from the six position sensors and the forces generated by six voice coil actuators, it is possible to control the position and angle of the floating element to any desired level. In the velocity mode, the coils B are used as actuators and the control system has sine wave reference trajectories for the position and angles. While the sensing element moves with the given trajectory, the voltages induced in the coils B are measured. The calibration coefficients for the 
force and torque measurements can be determined by dividing the amplitude of the induced voltages by the amplitude of the linear and angular velocities respectively. In the force/torque sensing mode, the coils A are used as actuator and the sensing element is controlled at a constant position and angle. External forces and torques actuating in the sensing element are compensated by the control system and can be determined by measuring the current flowing in the coils A. A 6 coil system that operates only in the force/torque sensing mode was already described in [10].

The system is designed to measure forces and torques in a range of $\pm 2 \mathrm{~N}$ and $\pm 0,1 \mathrm{Nm}$ respectively with a maximal relative expanded measurement uncertainty $(\mathrm{k}=2)$ of $10 \cdot 10^{-5}$. In order to achieve this uncertainty, all components of the system (figure 1, right hand side) have to fulfill specific requirements of stability and linearity and some of them also measurement traceability. This includes the voice coil actuators, the position sensors, the resistance standards, voltage measuring systems and time reference. In the following sections, more details for each component are provided with the respective requirements.

\subsection{Voice coil actuators}

The voice coil actuators used in this system are shown in Figure 2. They are composed by a magnet system made of $\mathrm{NeFeB}$ permanent magnets. The magnitude of the static force generated by each voice coil actuator is given by the following expression:

$$
F=k_{B l} i
$$

where $k_{B l}$ indicates the calibration factor of the voice coil actuator, which is proportional to the product of the magnetic flux density $B$ with the length of the conductor $l$. All voice coil actuators have a nominal value for $k_{B l}$ equal to $20 \mathrm{~N} / \mathrm{A}$. The magnitude of the torque generated by the voice coil actuators is given by:

$$
T=k_{d B l} i
$$

where $k_{d B l}$ is the calibration factor for the torque, which is proportional to $B, l$ and the distance $d$ between the axis of the coil and the torque center of the sensing element. The torque center is the reference point for the torque measurement and can be virtually fixed to any position. In our case it is defined in the geometric center of the sensing element. All voice coil actuators have a nominal value for $k_{d B l}$ equal to $1 \mathrm{~N} \cdot \mathrm{m} / \mathrm{A}$.

As described in [10], there are two voice coil actuators for each measurement direction, responsible for generating the respective force and torque components. For instance, the coils A-1 and A-2 generate the following static force and torque components:

$$
\begin{gathered}
F_{x}=F_{1}+F_{2}=k_{B l 1} i_{1}+k_{B l 2} i_{2} \\
T_{z}=T_{1}+T_{2}=-k_{d B l 1} i_{1}+k_{d B l 2} i_{2}
\end{gathered}
$$

These expressions are for the force/torque sensing mode, when the coils A compensate the external forces and torques applied to the sensing element. For the velocity mode the following expressions can be obtained:

$$
\begin{array}{rll}
u_{B l 1}=\hat{k}_{B l 1} v_{x} & \text { and } & u_{B l 2}=\hat{k}_{B l 2} v_{x} \\
u_{d B l 1}=\hat{k}_{d B l 1} \Omega_{z} & \text { and } & u_{d B l 2}=\hat{k}_{d B l 1} \Omega_{z}
\end{array}
$$

$u_{B l 1}$ and $u_{B l 2}$ represent the voltages induced in the coils A-1 and A-2 when the sensing element is moved along the $x$ direction with a velocity amplitude $v_{x} . u_{d B l 1}$ and $u_{d B l 2}$ are the voltages induced in the same coils when the sensing element is rotated with angular velocity amplitude $\Omega_{z}$. This rotation must be about an axis that intersects the torque center and is parallel to the $z$ direction.

The working principle of the sensor relies on the fact that $k_{B l k}=\hat{k}_{B l k}$ and $k_{d B l k}=\hat{k}_{d B l k}$, for $k=1, \ldots, 6$. In this way it is possible to determine the calibration coefficients $k_{B l k}$ and $k_{d B l k}$ of the force and torque sensor during the velocity mode and use them to obtain a traceable force and torque measurement during the force/torque sensing mode. However, there are several assumptions necessary for this principle to work. The magnitude of the magnetic flux generated 

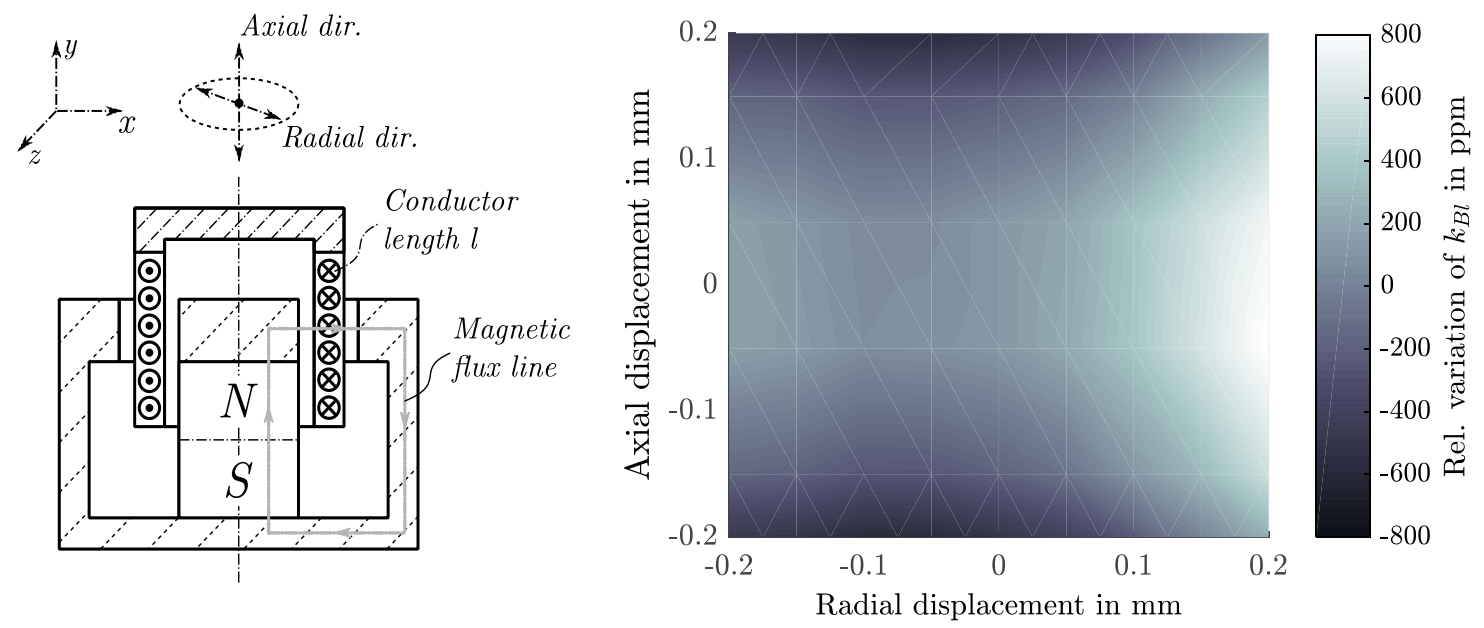

Figure 2: Voice coil actuator and $k_{B l}$ as a function of the position of the voice coil [9]

by the permanent magnets depend on the temperature of the magnetic material. For NeFeB magnets this dependence is about $-0,1 \% / \mathrm{K}$ according to manufacturer specifications. After determining the calibration coefficients during the velocity mode, the temperature of the permanent magnets must be monitored to avoid error influences originated by temperature variation. It is known that this influence can be minimized by using SmCo magnetic materials, which have a lower temperature coefficient. The calibration factors $k_{B l k}$ and $k_{d B l k}$ of the coils A depend on the relative position (figure 2, right hand side) and angle of the coil to the permanent magnet. It means that the position and angle of the sensing element must be the same for both velocity and force/torque sensing mode. The amplitude of the movement during the velocity mode must not be too high in order to avoid error effects. During the velocity mode, the sensing element has also to precisely follow the moving trajectories. The precise movement is assured by the position sensors, which are discussed in the next section.

\subsection{Position sensors}

The position measuring system used in the force/torque sensor is shown in the figure 3 (left hand side). It is composed by a LED light source, an aperture slit and a dual photodiode. The LED and the dual photodiode are fixed to the sensor frame and the aperture slit is attached to the sensing element. As the aperture slit moves in the measurement direction, the light intensity in each photodiode changes. The currents driven by the photodiodes are proportional to the light intensity. The position of the aperture slit in the measurement direction can be obtained by measuring the currents of both photodiodes and calculating the difference them. This difference is divided by the sum of the currents with the objective to compensate temperature effects in the LED that change its light intensity:

$$
y_{a l}=k_{p s} \frac{i_{u}-i_{l}}{i_{u}+i_{l}}
$$

where $i_{u}$ and $i_{l}$ represent the currents driven by the upper and lower photodiodes respectively and $k_{p s}$ represents the gain of the position sensor. In order to measure the three position coordinates and angles of the sensing element it is necessary to use six of these position sensors. There are several aspects of these position sensors that must be considered for consistently measuring the position and angles of the sensing element. These position sensors have a nonlinear characteristic curve and the gain $k_{p s}$ depend on the distance of the aperture slit to the dual photodiode. Figure 3 (right hand side) contains results of measurements performed to quantify these effects. The non-linearity of the characteristic curve is shown by $k_{p s}$ as a function of the position of the aperture slit in $y$ direction, i.e. measurement direction. The dependence 

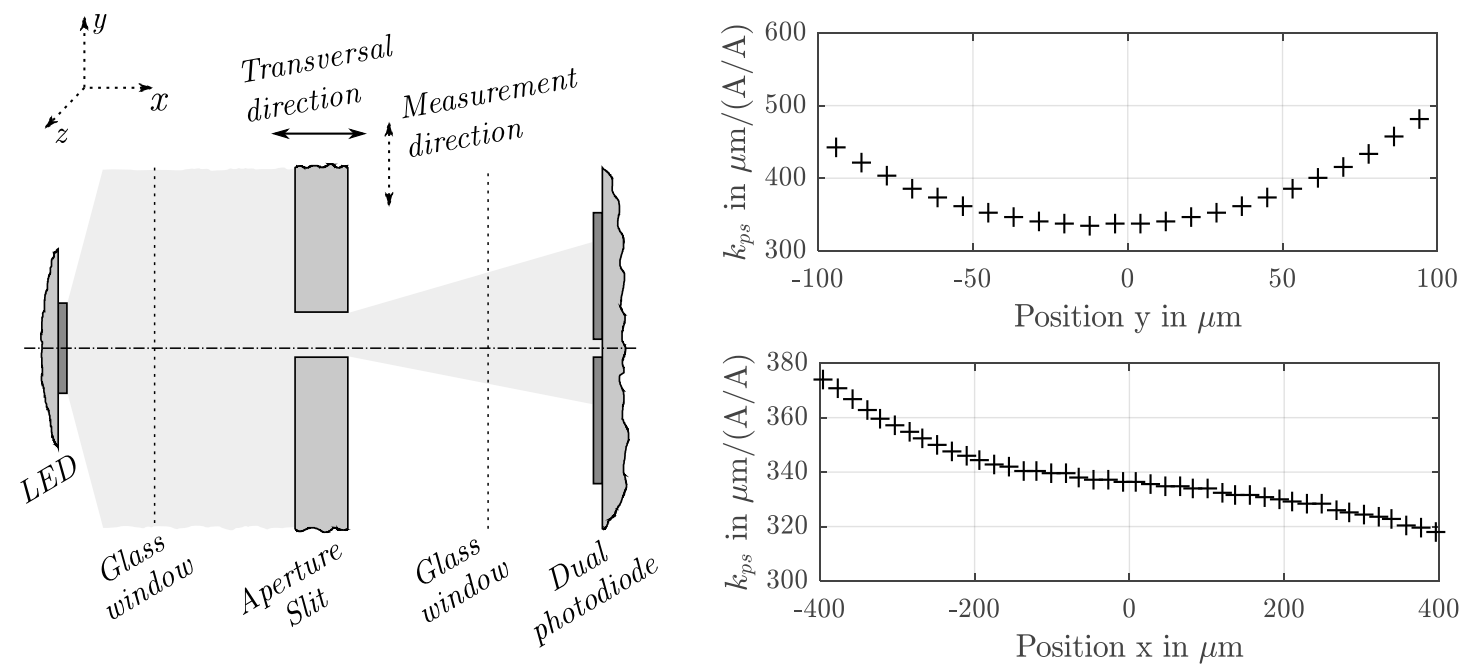

Figure 3: Position sensor and its gain in function of a displacement in the transversal direction [1]

of the gain $k_{p s}$ on the position in the $x$ direction, i.e. transversal direction, is shown in the same figure. There are also several uncertainties introduced due to assembly tolerances of the aperture slits, the LEDs and the photodiodes. In order to achieve the desired relative uncertainty for the force and torque measurement it is necessary to measure the position and angle amplitudes during the velocity mode with relative uncertainties smaller than $35 \cdot 10^{-6}$. The strategy used in this case is to calibrate the whole position and angle measuring system, which include the six individual position sensors, with a triple-beam laser interferometer (figure 4). This laser interferometer can be used to measure simultaneously a position coordinate and two angles of a mirror attached to the sensing element of the force/torque sensor. By sequentially mounting the sensor in three orthogonal orientations, all directions can be measured. Since the laser interferometer provides a traceable position and angle measurement, it is possible to calibrate the position and angle measuring system of the force/torque sensor and verify its stability. This step is fundamental in order to achieve the necessary positioning uncertainty.

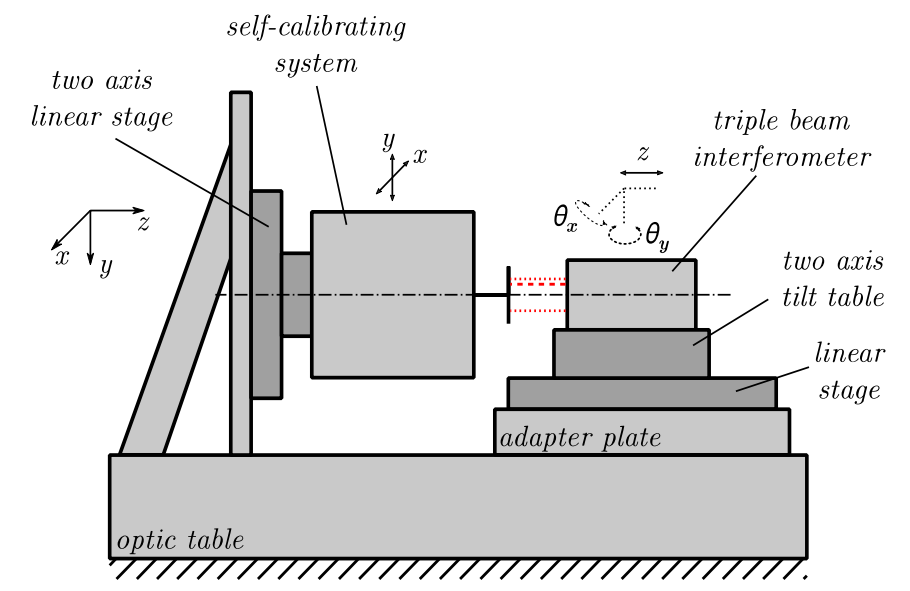

Figure 4: Calibration setup for the position and angle measuring system

\subsection{Voltage, time and resistance measurement}

The voltage, time and resistance traceable measurements are additional requirements for performing the self-calibration and measuring forces and torques with a relative uncertainty lower than $10 \cdot 10^{-5}$. During the velocity mode, the amplitude of the voltage induced in the voice coil actuators must be measured with an uncertainty lower than $1 \mu \mathrm{V}$. The amplitude of the linear velocity $v$ and the angular velocity $\Omega$ are obtained by measuring the amplitude of the position $x$ or angle $\theta$ and multiplying it by the angular frequency of the movement $\omega$ :

$$
v=x \omega, \quad \Omega=\theta \omega
$$


These expressions are only valid for a sinusoidal movement of the sensing element. That means the relative uncertainty of the velocity measurement is determined by the relative uncertainties of the position and frequency measurements. For this work, an angular frequency of $10 \cdot 2 \pi \mathrm{rad} / \mathrm{s}$ is expected to be measured with a relative uncertainty of $10 \cdot 10^{-6}$ by using a real-time digital controller. This uncertainty can be evaluated by comparing the time measurement of the digital controller with a traceable time reference.

During the force/torque sensing mode the current $i$ flowing in each voice coil actuator is determined by measuring the voltage drop $u$ in a reference resistor $R$ with a nominal resistance value of $10 \Omega$ :

$$
u=R i
$$

The voltage drop must be measured with an uncertainty of $20 \mu \mathrm{V}$ and the resistance must be known with a maximum relative uncertainty of $10 \cdot 10^{-6}$. The voltages are measured by using successive approximation analog-to-digital converter converters (ADC). These ADCs can be calibrated by using reference digital voltmeters (DVM) and a voltage source. With this experimental setup it is also possible to evaluate several other characteristics of the ADCs, like temperature sensitivity and long-term stability.

\section{MEASUREMENT UNCERTAINTY}

The relative expanded uncertainty for the force and torque measurements can be estimated by considering a simplified model with only one voice coil actuator. For the force measurement, the following equation can be obtained from (1), (8), (9) and assuming that the calibration factor $k_{B l}$ is equal to the ratio obtained during the velocity mode $u_{B l} / v$ :

$$
F=k_{B l} i=\frac{u_{B l}}{x \omega} \cdot \frac{u}{R}
$$

For the torque measurement, the following equation can be obtained from (2), (8), (9) and also assuming that the calibration factor $k_{d B l}$ is equal to the ratio obtained during the velocity mode $u_{d B l} / \Omega$ :

$$
T=k_{d B l} i=\frac{u_{d B l}}{\theta \omega} \cdot \frac{u}{R}
$$

Expressions for the combined measurement uncertainties of $F$ and $T$ can be obtained by using the method described in the guide to the expression of uncertainty in measurement [14]:

$$
\begin{aligned}
& \frac{\sigma_{F}^{2}}{F^{2}}=\frac{\sigma_{u_{B l}}^{2}}{u_{B l}^{2}}+\frac{\sigma_{u}^{2}}{u^{2}}+\frac{\sigma_{x}^{2}}{x^{2}}+\frac{\sigma_{\omega}^{2}}{\omega^{2}}+\frac{\sigma_{R}^{2}}{R^{2}} \\
& \frac{\sigma_{T}^{2}}{T^{2}}=\frac{\sigma_{u_{d B l}}^{2}}{u_{d B l}^{2}}+\frac{\sigma_{u}^{2}}{u^{2}}+\frac{\sigma_{\theta}^{2}}{\theta^{2}}+\frac{\sigma_{\omega}^{2}}{\omega^{2}}+\frac{\sigma_{R}^{2}}{R^{2}}
\end{aligned}
$$

Table 1: Measurement uncertainty contribuitions and combined measurement uncertainties

\begin{tabular}{ccccc}
\hline Variable & Description & Value & Uncertainty $\sigma$ & $\begin{array}{c}\text { Relative } \\
\text { uncertainty }\end{array}$ \\
\hline$u_{B l}, u_{d B l}$ & Induced voltage amplitude & $37 \mathrm{mV}$ & $1 \mu \mathrm{V}$ & $27 \cdot 10^{-6}$ \\
$u$ & Voltage drop in $R$ & $1 \mathrm{~V}$ & $20 \mu \mathrm{V}$ & $20 \cdot 10^{-6}$ \\
$x$ & Translation amplitude & $30 \mu \mathrm{m}$ & $1 \mathrm{~nm}$ & $33 \cdot 10^{-6}$ \\
$\theta$ & Rotation amplitude & $580 \mu \mathrm{rad}$ & $20 \mathrm{nrad}$ & $34 \cdot 10^{-6}$ \\
$\omega$ & Movement frequency & $10 \cdot 2 \pi \mathrm{rad} / \mathrm{s}$ & $100 \cdot 2 \pi \mu \mathrm{rad} / \mathrm{s}$ & $10 \cdot 10^{-6}$ \\
$R$ & Measurement resistor & $10 \Omega$ & $100 \mu \Omega$ & $10 \cdot 10^{-6}$ \\
\hline$F$ & Force measurement & $2 \mathrm{~N}$ & $98 \mu \mathrm{N}$ & $49 \cdot 10^{-6}$ \\
$T$ & Torque measurement & $0,1 \mathrm{~N} \cdot \mathrm{m}$ & $5,0 \mu \mathrm{N} \cdot \mathrm{m}$ & $50 \cdot 10^{-6}$ \\
\hline
\end{tabular}


Table 1 contains the single uncertainty contributions and the combined uncertainties for the force and torque measurements. A maximal relative expanded uncertainty $(\mathrm{k}=2)$ of $10 \cdot 10^{-5}$ is expected for the force and torque measurements in all components.

\section{FUTURE WORK}

The first step is to finish the implementation of this multi-component force/torque sensor and evaluate the measurement uncertainty for the forces and torques. After that, improvement possibilities for reducing the measurement uncertainty will be identified. This involves changing the voice coil actuators to have a more linear behavior and stable calibration factors, improving the linearity of the position measuring system, increasing the translation and rotation amplitudes during the velocity mode for reducing their relative uncertainties, increasing the magnitude of the calibration factors for reducing the relative uncertainty of the induced voltage, increasing the resistance of the measurement resistors for reducing the relative uncertainty of the voltage drop and using a reference clock with lower uncertainty for the time measurement. Additionally, the uncertainties for the dynamic force and torque measurement with this system will be analyzed as all.

\section{CONCLUSIONS}

The principle of a Kibble balance is being applied to the design of multi-component force and torque sensor with the objective to simplify the traceable multi-component measurement of forces and torques and improve the measurement uncertainty. With the system proposed here it is not necessary to derive the force quantity from the mass and gravity acceleration measurements. Instead, the forces and torques are derived from the measurement of voltages, positions, angles, resistances and time. In this way, the multi-component force and torque sensor can be calibrated in conditions where the local gravity acceleration $g$ is unknown or inexistent, as well as for any orientation relative to the $g$ direction. The system is also an alternative to the traditional calibration methods for force and torque sensors, which use deadweights. While the system shown here is being designed for measuring forces and torques with a relative uncertainty of $10 \cdot 10^{-5}$, traditional Kibble balance experiments are used to measure the gravitation force of weights with uncertainties of some $10^{-8}$, i.e. four orders of magnitude lower. This fact demonstrates the potential of the system shown here to reduce the measurement uncertainties in multi-component force and torque metrology.

\section{ACKNOWLEDGMENTS}

The authors are grateful to the German Science Foundation (Deutsche Forschungsgemeinschaft) for financial support of this work in the framework of the Research Training Group "Lorentz force velocimetry and Lorentz force eddy current testing".

\section{REFERENCES}

[1] C. Diethold, T. Fröhlich, F. Hilbrunner and G. Jäger, "High precission optical position sensor for electromagnetic force compensated balances", Proceedings of the IMEKO 21rd TC3 International Conference, IMEKO, 2010.

[2] C. Ferrero, Li Qing Zhong, C. Marinari, E. Martino, "New Automatic Multicomponent Calibration System with Crossed-Flexure Levers", Proceedings of the Third International Symposium on Measurement and Control in Robotics, 1993. 
[3] D. Haddad, F. Seifert, L. S. Chao, S. Li, D. B. Newell, J. R. Pratt, C. Williams, and S. Schlamminger, "Invited Article: A precise instrument to determine the Planck constant, and the future kilogram“, Review of Scientific Instruments, AIP Publishing, 87, 061301, 2016.

[4] D. Haddad, F. Seifert, L. S. Chao, A. Possolo, D. B. Newell, J. R. Pratt, C. J. Williams, and S. Schlamminger, "Measurement of the Planck constant at the National Institute of Standards and Technology from 2015 to 2017“, Metrologia, BIPM \& IOP Publishing Ltd, DOI: https://doi.org/10.1088/1681-7575/aa7bf2, 2017.

[5] F. Hilbrunner, H. Weis and T. Fröhlich, "Parameterization and optimisation of EMC balances based on the frequency response of the impedance", Measurement, Elsevier Science, Bd. 51, S. 349-355, 2014.

[6] G.-S. Kim, "The development of a six-component force/moment sensor testing machine and evaluation of its uncertainty", Meas. Sci. Technol., IOP Publishing Ltd, Volume 11, 1377-1382, 2000.

[7] J. Nitsche, S. Baumgarten, M. Petz, D. Röske, R. Kumme and R. Tutsch, "Measurement uncertainty evaluation of a hexapod-structured calibration device for multi-component force and moment sensors", Metrologia, BIPM \& IOP Publishing Ltd, Volume 54, 171$183,2017$.

[8] J. Schleichert, "Entwicklung und Untersuchung von Mehrkomponentensensoren für Kraft und Drehmoment", Ph.D. Thesis, Technische Universität Ilmenau, 2015.

[9] J. Schleichert, M. Carlstedt, R. R. Marangoni, I. Rahneberg and T. Fröhlich, "Dynamische Charakterisierung eines Dreikomponenten Kraftsensors mit Hilfe eines LorentzkraftLastwechslers“, Technisches Messen, Walter de Gruyter, 83(7-8), 430-438, 2016.

[10] J. Schleichert, I. Rahneberg, F. Hilbrunner and T. Fröhlich, “ Mehrkomponenten-Kraftund -Drehmomentsensor nach dem Prinzip der elektromagnetischen Kompensation", Technisches Messen, Walter de Gruyter, 83(7-8): 445-452, 2016.

[11] R. S. Davis, "What Is a Kilogram in the Revised International System of Units (SI)?“, J. Chem. Educ., ACS Publications, Inc, 92 (10), pp 1604-1609, 2015.

[12] S. Baumgarten, H. Kahmann and D. Röske, "Metrological characterization of a $2 \mathrm{kN} \cdot \mathrm{m}$ torque standard machine for superposition with axial forces up to $1 \mathrm{MN}$ ", Metrologia, BIPM \& IOP Publishing Ltd, Volume 53, 1165-1176, 2017.

[13] T. Fröhlich, I. Rahneberg, F. Hilbrunner, J. Schleichert, "Vorrichtung und Verfahren zur Kalibrierung von Kraft- und Drehmomentmesseinrichtungen", German Patent and Trade Mark Office, DE102016010668B3, 2017.

[14] Working Group 1 of the Joint Committee for Guides in Metrology, "Evaluation of measurement data - Guide to the expression of uncertainty in measurement", BIPM, 2008.

\section{CONTACTS}

R. R. Marangoni

Dr.-Ing. J. Schleichert

Dr.-Ing. I. Rahneberg

Dr.-Ing. F. Hilbrunner

Prof. Dr.-Ing. habil. T. Fröhlich rafael.marangoni@tu-ilmenau.de

jan.schleichert@tu-ilmenau.de

ilko.rahneberg@sios.de

falko.hilbrunner@sartorius.com

thomas.froehlich@tu-ilmenau.de 